\title{
Femtocell network power control scheme based on the weighted voting game
}

\author{
Sungwook Kim
}

\begin{abstract}
Future wireless networks are designed to cope with drastically increasing user demands. However, network resources reach the limits of their capacity to user requirements. Recently, femtocell networks have attracted much attention to enhance the efficiency of wireless resource usage. In this article, a new adaptive power control scheme is developed for the femtocell network system. By using the concept of the weighted voting game, the proposed scheme adaptively adjusts a transmit power level while ensuring relevant tradeoff between system efficiency and fairness. This power control paradigm can provide the ability to practically respond to current communication conditions and suitable for real network operations. Simulation results are presented to verify the effectiveness of the proposed voting game-based scheme in comparison with the existing schemes.
\end{abstract}

Keywords: Femtocell networks, Game theory, Weighted voting model, Power control, Fairness, Resource allocation problem

\section{Introduction}

The emergence of new wireless technologies is expected to support the remarkable growth in the number of users. However, in spite of high network infrastructures, wireless bandwidth is a particularly scare resource, especially when large amounts of data are required. A promising approach for the efficient wireless bandwidth usage is through the cellular technology, which has greatly enhanced the network capability. In cellular networks, the capacity of a wireless link has increased by getting the transmitter and receiver closer to each other. Therefore, it is necessary to reduce cell sizes and transmit distance to improve cellular capacity $[1,2]$.

The recent concept of femtocells offers an economically viable solution to achieving high cellular capacity and improved coverage. A femtocell is a small indoor area covered by a low-power access point, referred to as femtocell access point (FAP). Usually, FAPs are situated around high user density hotspots to improve communication capacity. Therefore, the deployment of femtocell structure comprises a conventional cellular network plus embedded femtocell hotspots. The most important advantages of femtocell are very little upfront cost to

Correspondence: swkim01@sogang.ac.kr

Department of Computer Science, Sogang University, 35 Baekbeom-ro (Sinsu-dong), Mapo-gu, Seoul 121-742, South Korea offload huge traffic from the expensive cellular networks and the ability to achieve high data rate in some places where the macrocell cannot provide signals with high quality. It is very meaningful in future wireless communication networks [1-3].

Key issues of femtocell operations are the design of effective power control protocols, which significantly influence the wireless network performance [3]. In addition, the limited wireless bandwidth has to be shared fairly among several users. Fair resource assignment is another prominent factor for the femtocell management $[4,5]$. Due to these reasons, the development of adaptive strategies for efficient power control and fair bandwidth assignment algorithms plays a critical role in determining overall network performance. However, it is a complex and difficult work under a dynamically changing network environment.

In recent years, algorithmic game theory has received a lot of attention in field of telecommunications. The reason for this interest is that game theory focuses on the research of intelligent game players, who can compete, cooperate, and negotiate for their benefits. In telecommunication domains, network agents are selfish and built to maximize their payoffs without requiring human interventions. Therefore, it can be modeled by means of game theory [6]. Cooperative game, also called coalition game, is a branch of 
game theory to deal with multi-person decision-making situations. In a cooperative situation, the basic assumption is that players make binding commitments and find it beneficial to cooperate in the game. Therefore, the main interest is to fairly distribute the outcome to each player according to their contributions. Especially, the operations contending for network resources rely on the cooperation of the each network agents. Therefore, cooperation game models are widely used for wireless network management algorithms $[7,8]$.

Weighted voting game (WVG) is a well-known cooperative game model and a lot of researches have been carried out in political science, economics, logic theory, and distributed systems. In such games, each player has a weight, and a coalition of players wins the game if its total weight exceeds a certain quota. Therefore, the WVG model can formulate a decision-making process that is designed to give different amounts of influence to different members $[6,9,10]$. In femtocell networks, network agents must take a joint decision leading to a certain outcome, which may have a different impact on each of the agents. Therefore, voting-based decisionmaking procedure is an attracting model for the femtocell network management.

Inspired by the preceding discussion, we propose a new femtocell network power control scheme based on the WVG. In the proposed scheme, each mobile user is assigned a non-negative weight and makes a vote in favor of a specific femtocell base station. If the total weight of those voting is equal to or greater than the pre-defined value, that femtocell becomes to be a winner and can control its downlink power level. However, this efficientoriented approach may lead to throughput unfairness. To avoid this unfairness problem, we characterize the concept of fairness, which is considered explicitly at the design stage of power control algorithm. Therefore, power and bandwidth in the winning femtocell are dynamically adjusted to provide a balanced solution. Based on the selfadaptability and real-time effectiveness, the main novelty of the proposed scheme is the ability to ensure relevant tradeoff between efficiency and fairness.

Many literatures and the Small Cell Forum (www. smallcellforum.org) support the wide-scale adoption of femtocell. Lopez-Perez et al. [11] studied a downlink case for WiMAX femtocell networks. Valcarce et al. [12] applied a finite-difference time domain method to predict the coverage of WiMAX femtocells. Lopez-Perez et al. [13] used a centralized method of dynamic frequency planning to minimize the overall femtocell network interference to allocate the bandwidth to femtocells. Sundaresan and Rangarajan [14] studied the resource management problem in orthogonal frequency-division multiple access (OFDMA) femtocells and proposed a location-based allocation scheme between macrocells and femtocells to adapt the varying user population. In [15], resource sharing and access control in OFDMA femtocell networks are studied, where users' selfish characteristics are considered and an incentive mechanism is designed for subscribers to share the resource of femtocell base stations. In [16], the problem of cross-tier interference in autonomous femtocell networks is studied by using cognitive radio technology to realize the cognitive radio resource management. And a strategic game is proposed to implement the interference mitigation. The interference management for long-term evolution (LTE) networks with femtocells using cognitive radio technology is investigated in [17]. Then based on a distributed architecture for LTE networks, the authors recommend to use two game theoretical mechanisms to mitigate the cochannel interference. In [18], a distributed power control algorithm is proposed that enables users to eventually achieve their fixed target SINRs. As long as all the SINR targets are feasible, this iterative algorithm converges to a Pareto-optimal solution at a minimal aggregate transmit power.

Recently, two interesting femtocell power control schemes have been presented. The Power Control in Overlay Femtocell (PCOF) scheme in [19] can properly control the quality of service (QoS) in the femtocell users. With feasibility conditions, new joint power control and dynamic channel re-allocation procedures are suggested such that the QoS of users was ensured all the time. In the PCOF scheme, the fundamental capacity limitation of spatial bandwidth sharing among a macrocell user and a femtocell user is identified. In addition, a downlink power control problem is formulated to address the co-channel interference, as well as provide QoS to both the macrocell user and the femtocell users. Therefore, the PCOF scheme is a joint power control, channel management, and admission control procedure such that the priority of the macrocell users is always ensured. The Game Theoretic Power Control (GTPC) scheme [20] is proposed as a power control algorithm by using a non-cooperative game model. This scheme formulates a payoff function to provide fairness and minimize interference by considering loads of individual femtocells. In addition, the GTPC scheme proves that this payoff function can be appeared as a supermodular type. The GTPC scheme can be applied to the decentralized environment, and leads transmission power to reach a steady-state condition, i.e., Nash equilibrium. Through mathematical analysis and numerical results, the authors show that the GTPC scheme has several good characteristics. All the earlier works have attracted a lot of attention and introduced unique challenges. Compared to these schemes $[19,20]$, the proposed scheme attains better performance for femtocell network systems. 
The remainder of this article is organized as follows. Section 2 presents the proposed algorithms in detail. In Section 3, performance evaluation results are presented along with comparisons with the PCOF and GTPC schemes proposed in $[19,20]$. Through simulation, we show the ability of proposed scheme to achieve high accuracy and promptness in dynamic network environments. Finally, concluding remarks are given in Section 4.

\section{Proposed power control algorithm}

In this section, we examine the applicability of WVG to design a power control algorithm, and the proposed scheme is explained in detail. The proposed scheme is designed based on the WVG and can provide a wellbalanced network performance under rapidly changing network environments.

\subsection{WVG-based selection algorithm}

In cooperative games with a finite set $(N)$ of players, a function $v$ is defined as $v: 2^{N} \rightarrow \mathbb{R}$, such that $(\varnothing)=0$ and $v(M) \leq v(N)$ whenever $M \subseteq N$. It is based on the assumption that each player should decide whether he/she join a coalition or not. An important subclass of cooperation games is the WVG, which has been applied in various political and economic organizations for structural or constitutional purposes. A WVG is represented by the voting body $\left[q ; \omega_{1}, \ldots, \omega_{n}\right]$ where $\omega_{i}$ represents the voting weight of player $i$ and $q\left(0<\omega_{i}<q\right)$ is the quota needed to win. All the weights and quota are positive real numbers. If $\mathcal{W}(\mathbb{S}) \geq q$ where $(\mathbb{S})=\sum_{i \in \mathbb{S}} \omega_{i}$, a coalition $S$ of players $(\mathbb{S} \subseteq N)$ is winner and $v(\mathbb{S})=1$. Otherwise, it is loser and $v(\mathbb{S})=0$. Therefore, $v(\mathbb{S}) \in\{0,1\}$ for any $\mathrm{S}$ and if $\mathbb{S} \subseteq T \subseteq$ $N$ then $v(\mathbb{S}) \leq v(T)[9,10]$.

In this article, we develop a WVG model for users in femtocell networks. To design the WVG, the questions to be answered are what the weights and quota should be, how to calculate outcomes of a weighted vote, and finding the effective distribution of the resulting outcome from their joint action [6,9]. Recently, voting game approaches have been studied well. However, existing approaches only concerned and focused on theoretical modeling and mathematical analysis. Therefore, practical implementation issues are not yet well developed [10]. From a fundamental viewpoint, the proposed scheme places more emphasis on the practical implementation in real world network operations.

In a dense traffic load area, a lot of FAPs are deployed within small coverage area. Therefore, users can receive many signals from the several neighbor FAPs. Under the multi-FAPs environment, each user is responsible for selecting a FAP to maximize its own payoff. Usually, the main interest of each user is to maximize the amount of transmitted data with the lower energy consumption. However, there is a fundamental tradeoff. To capture this conflicting relationship, a utility function is defined by considering the ratio of the throughput to transmit power $[21,22]$. In the proposed game model, the utility function for the $i$ th user with $k$ th FAP $\left(U_{i}^{k}\right)$ is defined as follows:

$$
U_{i}^{k}(\mathbb{P})=\frac{T_{i}^{k}(\mathbb{P})}{u_{p_{i}}}, \text { s.t. } . p_{i} \in\left\{u_{p_{\min }} \ldots u_{p_{\max }}\right\} 1 \leq i \leq n \text { and } 1 \leq k \leq m,
$$

where $u \_p_{\min }$ and $u \_p_{\max }$ are the pre-defined minimum and maximum uplink power levels. $n$ and $m$ are the number of macrocell users and FAPs, respectively. $u \_p i$ is the uplink power level of $i$ th user and $P$ is the transmit uplink power vector. $T_{i}^{k}(\mathbb{P})$ is the throughput of $i$ th user by using $k$ th FAP. Usually, throughput is defined as the number of information bits that are transmitted without error per unit-time. In wireless communications, it can be achieved with the SINR in the effective range $[21,22]$. Therefore, to estimate the throughput of each user, his SINR should be obtained first. A general formula for the $i$ th user's SINR from the $k$ th FAP $\left(\gamma_{i}^{k}(\mathbb{P})\right)$ is defined as follows $[21,22]$ :

$$
\gamma_{i}^{k}(\mathbb{P})=\frac{u \_p_{k} h_{i k}}{\sigma_{i}+\sum_{t \neq k} u \_p_{t} h_{i t}}, \text { s.t., } 1 \leq t \leq m,
$$

where $\sigma_{i}$ is the background noise power, which is typically fixed value. $h_{i k}\left(o r h_{i t}\right)$ refers to the $i$ th user's path gain from the $k$ th (or th) FAP. In this article, we follow the assumption in [22]; users' device transmitters use variablerate M-QAM, with a bounded probability of symbol error and trellis coding with a nominal coding gain. Therefore, the uplink throughput of the $i$ th user from the $k$ th FAP $\left(T_{i}^{k}(\mathbb{P})\right)$ can be expressed as

$$
T_{i}^{k}(\mathbb{P})=W_{i} \times \log _{2}\left(1+\frac{\gamma_{i}^{k}(\mathbb{P})}{\Omega}\right)
$$

where $W_{i}$ is the assigned channel bandwidth in the $i$ th user, and $\Omega(\Omega \geq 1)$ is the gap between uncoded M-QAM and the capacity, minus the coding gain [22]. By combining (1) with (3), the utility function for the $i$ th user from the $k$ th FAP is defined and parameterized as follows [22]:

$$
U_{i}^{k}\left(u \_p_{i}, \mathbb{P}_{-i}\right)=\frac{1}{u \_p_{i}} \times\left[W_{i} \times \log _{2}\left(1+\gamma_{i}^{k}(\mathbb{P}) / \Omega\right)\right],
$$

where $\mathbb{P}_{-i}$ is the uplink transmit power vector without the user $i$. In a distributed self-regarding fashion, each individual user in the femtocell network is independently 
interested in the sole goal of maximizing his utility function. Finally, the $i$ th user's payoff is defined as follows:

$$
\begin{aligned}
U_{i}= & \max _{\begin{array}{c}
u \_p_{i} \\
\text { and } k
\end{array}} U_{i}^{k}\left(u \_p_{i}, \mathbb{P}_{-i}\right) \\
= & \max _{\substack{u \_p_{i} \\
\text { and } k}} \frac{1}{u \_p_{i}} \times\left[W_{i} \times \log _{2}\left(1+\frac{\gamma_{i}^{k}(\mathbb{P})}{\Omega}\right) .\right.
\end{aligned}
$$

To maximize the network throughput, each user's received payoff based on the utility function is a critical factor. In this article, we shall assume that each voter's weight is defined as his payoff $\left(\omega_{i}=U_{i}\right)$ and the quota $(Q)$ needed to win is defined as follows:

$$
Q=\rho \times \sum_{i \in N} \omega_{i}, \text { s.t., } \rho_{\min } \leq \rho \leq 1
$$

where $\rho$ is a decision factor for the quota. Under dynamically changing environments, a fixed value of $\rho$ cannot effectively adapt to the current network condition. Therefore, the $\rho$ value should dynamically be adjustable. In order to implement the $\rho$ value adjustment, we partition the time-axis into equal intervals of length unit_time, and the parameter $\rho$ can dynamically be decreased (or increased) by $\Delta \rho$. The value of $\rho\left(\rho_{\min } \leq\right.$ $\rho \leq 1)$ is multiples of $\Delta \rho$, and we set $\Delta \rho=0.1$ in this study. When a winner FAP is decided, the current $\rho$ value is increased by $\Delta \rho$. If no FAP becomes to be a winner during the predefined time period $\left(T_{-} P\right)$, the current $\rho$ value is determined as $\rho=\min \left(\rho-\Delta \rho, \rho_{\min }\right)$.

\subsection{Fairness-based resource control algorithm}

Fairness is a prominent issue for the network management. However, fairness-oriented control approaches may lead to a system inefficiency, which degrades total network performance quite seriously [4]. Therefore, the most proper combination of the efficiency and fairness requirements is the major issue. In this article, the fairness is defined as an equitable transmission rate for different individual users. To characterize the fairness notion, we follow the Jain's fairness index [4], which has frequently been used to measure the fairness of network resource allocations. The fairness among users in the winning coalition $S\left(F_{\mathbb{S}}\right)$ is defined as follows:

$$
F_{\mathbb{S}}=\frac{\left(\sum_{i \in \mathbb{S}} T_{i}(\mathbb{P})\right)^{2}}{|\mathbb{S}| \times \sum_{i \in \mathbb{S}}\left(T_{i}(\mathbb{P})\right)^{2}}, \text { s.t., } \sum_{i \in \mathbb{S}} \omega_{i} \geq Q \text {. }
$$

The range of $F_{\mathbb{S}}$ is varied from 0 to 1 . To improve the $F_{\mathbb{S}}$, the channel bandwidth for each user $(W)$ is adjusted. For the fair-bandwidth allocation, the most common idea is max-min fairness concept [5]. It aims at allocating as much as possible to poor users, while not unnecessarily wasting resources. However, to achieve max-min fair rates, global information is needed. Therefore, this approach requires huge exchange of information [5]. In this article, based on the max-min fairness notion, we propose a practical protocol to implement fair-based resource allocation in the winning coalition $S$. The main goal of the proposed algorithm is to distribute bandwidth for each user's communication as evenly as possible, without unduly reducing system efficiency. First, the minimum bandwidth allocation amount $\left(M \_a\right)$ is estimated by the using fairness index $\left(F_{\mathbb{S}}\right)$. And then, additional allocation amount $\left(A \_a\right)$ for each user is defined according to each throughput. The assigned bandwidth for the user $i W_{i, i \in \mathbb{S}}$ is calculated as follows:

$$
\begin{aligned}
& W_{i}=M_{-} a+A_{-} a_{i} \\
& \text { s.t., } M_{a}={ }^{\left(F_{\mathbb{S}} \times T W_{\mathbb{S}}\right)} /{ }_{n_{\mathbb{S}}}, A_{a_{k}} \\
&=\eta \times\left[T W_{\mathbb{S}}-\left(F_{\mathbb{S}} \times T W_{\mathbb{S}}\right)\right]=\text { and } \eta \\
&=\frac{1}{T_{i}(\mathbb{P})} / \sum_{j=1}^{n_{\mathbb{S}}}\left(\frac{1}{T_{j}(\mathbb{P})}\right)
\end{aligned}
$$

where $T W_{\mathbb{S}}, n_{\mathbb{S}}$ are the total bandwidth amount and the number of users in the coalition $S$, respectively. $A \_a_{i}$ is an additional allocation for the user $i$ and $\eta$ is a control factors for the inverse weighted allocation. According to (8), the channel bandwidth adjustment continues until all the users' bandwidths in $S$ are re-assigned.

\subsection{Multi-objective utility function for efficiency and fairness}

The proposed voting game model employs the doubledecision rule in which two conditions must be satisfied in terms of efficiency and fairness. During the voting game, a coalition $S$ is winning if $\sum_{i \in \mathbb{S}} \omega_{i} \geq Q$. And then, the winning FAP can select its downlink power level $\left(d_{\_} p_{\mathbb{S}}\right)$, which is adjusted within the pre-defined downlink power levels (i.e., $\_p_{\mathbb{S}} \in\left\{d_{p_{\min }} \ldots d_{-} p_{\max }\right\}$ ). To decide the downlink power level, we consider not only the system efficiency, but also the concept of fairness. To characterize the concept of efficiency, the efficiency index $(E)$ is defined as follows:

$$
E_{\mathbb{S}}=\frac{\sum_{i \in \mathbb{S}} U_{i}^{d}\left(d \_p_{\mathbb{S}}\right)}{|\mathbb{S}| \times\left(\max _{i} U_{i}^{d}\left(d \_p_{\mathbb{S}}\right)\right)}, \text { s.t., } \sum_{i \in \mathbb{S}} \omega_{i} \geq Q
$$

where $U_{i}^{d}\left(d_{p_{\mathrm{S}}}\right)$ is the user $i$ 's downlink utility with $d_{p \mathrm{~s}}$ power level. To deal with this efficiency-fairness combination control problem, a multi-objective utility function $\left(M_{-} E F\right)$ is developed based on the weighted sum method. 
By using dynamic joint operations, the proposed multiobjective utility function $\left(M_{-} E F\right)$ is formulated as follows:

$$
M_{-} E F=\left[\beta \times E_{\mathbb{S}}\right]+\left[(1-\beta) \times F_{\mathbb{S}}\right]
$$

where

$$
\beta=\frac{\left(\sum_{i=1}^{n} T_{i}(\mathbb{P})\right)^{2}}{n \times \sum_{i=1}^{n}\left(T_{i}(\mathbb{P})\right)^{2}}
$$

where $\beta$ controls the relative weights given to efficiency and fairness. By choosing a different weight value, the preference of the decision-maker is taken into account. Under diverse network environments, we treat it as an online decision problem and adaptively modify $\beta$ value. When the fairness of all users in a macrocell is high, we can put more emphasis on the system efficiency. In this case, a higher value of $\beta$ is more suitable. When the system fairness is low, the FAP's power control decision should strongly depend on the fairness. In this case, a lower value of $\beta$ is more suitable for system fairness. In the online algorithm, the value of $\beta$ is dynamically adjusted based on the total system fairness (i.e., $F_{N}$ : the fairness index of all users in a macrocell). By the real-time network monitoring, the system can be more responsive to current network conditions. Finally, the winning FAP's downlink power level $\left(P_{\mathbb{S}}\right)$ is decided as follows:

$$
\begin{aligned}
& d_{-} P_{\mathbb{S}}=\max _{d_{-} P_{\mathbb{S}}}\left(M M_{-} E F\right) \text { s.t., } d_{-} P_{\min } \leq d_{-} P_{\mathbb{S}} \leq d_{-} P_{\max } \\
&=\max _{d_{-} P_{\mathbb{S}}}\left[\beta \times \frac{\sum_{i \in \mathbb{S}} U_{i}^{d}\left(d_{-} p_{\mathbb{S}}\right)}{|\mathbb{S}| \times\left(\max _{i} U_{i}^{d}\left(d_{-} p_{\mathbb{S}}\right)\right)}\right] \\
&+\left[(1-\beta) \times \frac{\left(\sum_{i \in \mathbb{S}} T_{i}(\mathbb{P})\right)^{2}}{|\mathbb{S}| \times \sum_{i \in \mathbb{S}}\left(T_{i}(\mathbb{P})\right)^{2}}\right]
\end{aligned}
$$

\subsection{The main steps of proposed algorithm}

The main goal of proposed scheme is to ensure relevant tradeoff between efficiency and fairness. Even though the fairness-related solution concepts (e.g., the Shapley value) are studied well, the computational complexity makes it practically unable to implement in the realistic network operation model. To maximize implementation practicality, we would employ distributed and dynamic online game methodologies to develop a power control scheme. In general, due to heavy control and implementation overheads, centralized control approaches are impractical methods. But, a distributed mechanism can transfer the computational burden from a central system to the distributed nodes. In the proposed algorithm, users make their control decisions in a self-regarding distributed fashion. This distributed approach can dramatically reduce the computational complexity and overheads comparing to the traditional static centralized approach. Usually, the traditional optimal and centric algorithms have exponential time complexity. However, the proposed scheme has polynomial time complexity for power control decisions. Therefore, the proposed scheme can provide a good tradeoff between the practical implementation complexity and the optimized network performance.

In the proposed algorithm, the result of FAP's decision is the input back to users. At the end of each game's iteration, users dynamically re-estimate their decisions. This feedback-based repeated process can analyze strategic decisions and capture each user's behavior, which is defined as a power control algorithm. After a finite number of rounds, users' decisions can reach an efficient network solution. The main steps of the proposed power control algorithm are given next.

Step 1: At the initial game iteration, each mobile user dynamically selects the uplink power level $\left(u_{-} p\right)$ and the most adaptable FAP to maximize his payoff according to (5).

Step 2: If a FAP's weighted voting sum is larger than the current quota $\left(\sum_{i \in \mathbb{S}} \omega_{i}>Q\right)$, this FAP becomes to be a winner.

Step 3: For the coalition $S$ of winning FAP, $F_{\mathbb{S}}$ and $M_{-} a$ are estimated based on (7) and (8), respectively. Step 4: By using the bandwidth adjustment procedure, each user's channel bandwidth $(W)$ in $S$ is adjusted according to (8).

Step 5: According to (11), the winning FAP selects the best downlink power level to maximize the multiobjective payoff.

Step 6: The result of FAP's decision is the input back to users. At the end of each game's iteration, users iteratively adapt their decisions (i.e., uplink power level and FAP selection) to maximize their payoffs. This approach can analyze the current strategic decision in a distributed online manner.

Step 7: Every unit_time, the $\rho$ and $Q$ values are examined and adjusted periodically.

Step 8: By using the dynamics of feedback-based repeated process, FAPs and users can be interacting with one another and make their decisions in a way to reach an efficient network solution.

\section{Performance evaluation}

In this section, the effectiveness of the proposed scheme is validated through simulation; a simulation model is proposed for the performance evaluation. With a simulation study, the performance superiority of the proposed 
scheme can be confirmed. The assumptions implemented in the simulation model were as follows:

- The simulated system is assumed as a CDMA system with one macro cell.

- 3 FAPs and 20 mobile users are distributed randomly over the cell area.

- The capacity of network bandwidth is $30 \mathrm{Mbps}$.

- Network performance measures obtained on the basis of 50 simulation runs are plotted as a function of network operation time.

- The durations of call services are exponentially distributed.

- For simplicity, we assume the absence of physical obstacles in the experiments.

Table 1 shows the system parameters used in the simulation. In order to emulate a real network system and for a fair comparison, we used the system parameters for a realistic simulation model [23].

Performance measures obtained through simulation are system throughput, normalized user utility, network fairness, etc. In this article, we compare the performance of the proposed scheme with existing schemes-the PCOF scheme [19] and GTPC scheme [20]. Even though these existing schemes dynamically decide the transmitting power level for wireless networks, there are several disadvantages. First, these existing schemes cannot adaptively estimate the current network conditions. In addition, these schemes operate the network system by the fixed control parameters. Under dynamic network environments, control mechanisms by using static thresholds can cause potential erroneous decisions.

In Figures 1, 2, 3, and 4, the $x$-axis (a horizontal line) marks the time lag. In order to implement the time-driven simulation, we partition the time-axis into equal intervals of length unit_time. The $y$-axis (a vertical line) represents the normalized value for each performance criteria.

Figure 1 compares the performance of each scheme in terms of the system throughput. It is measured as a normalized serviced data amount. From the network providers' point of view, it is a very important factor. It is shown that under initial operation time, the system throughput is virtually the same for all the schemes. However, as the network operation time increases, the system throughput of proposed scheme is better than the other schemes. Due to the inclusion of the adaptive online feedback approach, we can observe that the proposed scheme gains the better throughput.

Figure 2 shows the comparison of the normalized user payoff. All the schemes have similar trends. However, the feature of dynamic game-based model can ensure the excellent user's payoff under dynamic environment situations. Therefore, the proposed scheme can effectively adapt to the current wireless network condition while providing the better performance than other schemes.

Figure 3 indicates the fairness of macrocell network. In this article, the macrocell network fairness $\left(N_{\mathrm{F}}\right)$ is defined as the fairness among total users in the macrocell network like as

$$
N_{F}=\frac{\left(\sum_{i=1}^{n} T_{i}(\mathbb{P})\right)^{2}}{n \times \sum_{i=1}^{n}\left(T_{i}(\mathbb{P})\right)^{2}}
$$

where $n$ is total number of users in the macrocell network. $N_{\mathrm{F}}$ is the same as the $\beta$ value in (10). An intelligent fairness policy, like the proposed scheme, makes the system adaptable to achieve an excellent fairness in the macrocell network. Therefore, the proposed scheme can maintain a significant better fairness than the other existing schemes. It is highly desirable for the operation of multi-user system.

Table 1 System parameters used in the simulation experiments

\begin{tabular}{|c|c|c|c|}
\hline Parameter & Value & \multicolumn{2}{|l|}{ Description } \\
\hline$n$ & 20 & \multicolumn{2}{|c|}{ Number of wireless mobile users } \\
\hline$m$ & 3 & \multicolumn{2}{|l|}{ Number of FAPs } \\
\hline$\Delta \rho$ & 0.1 & \multicolumn{2}{|l|}{ Control factor to adjust $\rho$ value } \\
\hline$T \_P$ & 10 unit_time & \multicolumn{2}{|c|}{ The predefined time period to control $\rho$ value } \\
\hline$\rho_{\min }$ & $1 / \mathrm{m}$ & \multicolumn{2}{|l|}{ Pre-defined minimum $\rho$ value } \\
\hline$u \_p_{\min }, u \_p_{\max }$ & $50 \mathrm{~mW}, 100 \mathrm{~mW}$ & \multicolumn{2}{|c|}{ pre-defined minimum and maximum uplink power levels } \\
\hline$d \_p_{\min }, d \_p_{\max }$ & $50 \mathrm{~mW}, 100 \mathrm{~mW}$ & \multicolumn{2}{|c|}{ Pre-defined minimum and maximum downlink power levels } \\
\hline unit_time & $1 \mathrm{~s}$ & \multicolumn{2}{|c|}{ Equal intervals of time-axis length } \\
\hline M & $1 \ldots 6$ & \multicolumn{2}{|c|}{ Number of power levels (strategies) } \\
\hline$\sigma$ & $1 \times 10^{-10}$ & \multicolumn{2}{|c|}{ Background noise power in wireless communications } \\
\hline$\beta$ & $0 \leq \beta \leq 1$ & \multicolumn{2}{|c|}{ Control factor for the relative efficiency and fairness weight } \\
\hline Parameter & Initial & Description & Values \\
\hline$u \_p, d \_p$ & $50 \mathrm{~mW}$ & Uplink, downlink power level & $50,60,70,80,90,100 \mathrm{~mW}$ \\
\hline
\end{tabular}




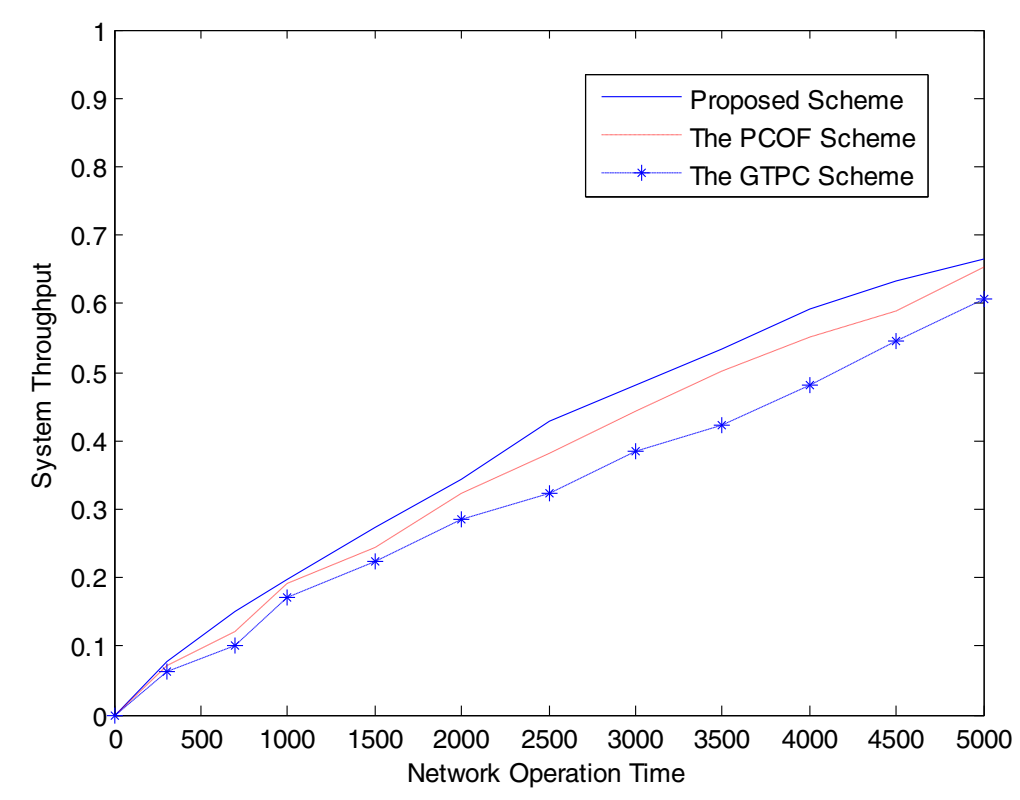

Figure 1 System throughput.

The curves in Figure 4 present the fairness in the FAP, which is also obtained according to the formulae of (12). However, in this case, $n$ is the number of users in the FAP. Due to the inclusion of the fairness-oriented control algorithm, we also observe that the proposed scheme can gain the higher FAP fairness than the PCOF and GTPC schemes.

The simulation results shown in Figures 1, 2, 3, and 4 demonstrate that the proposed scheme generally exhibits better performance compared with the other existing schemes. Based on the adaptive real-time monitoring, the proposed algorithm is flexible, adaptable, and able to sense the dynamic changing network environment. This feature leads to a balance appropriate network performance while other schemes $[19,20]$ cannot offer such an attractive network performance.

\section{Summary and conclusions}

The femtocellular network is one of the most promising future network technologies to meet the demand of the

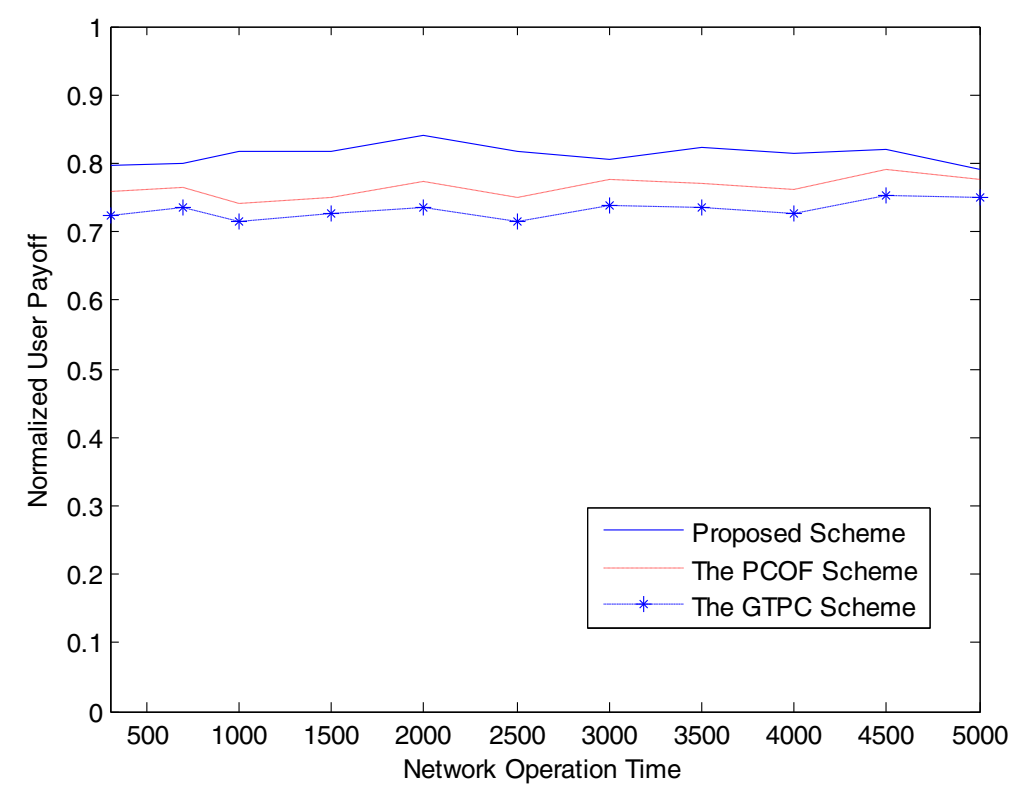

Figure 2 Normalized user payoff. 


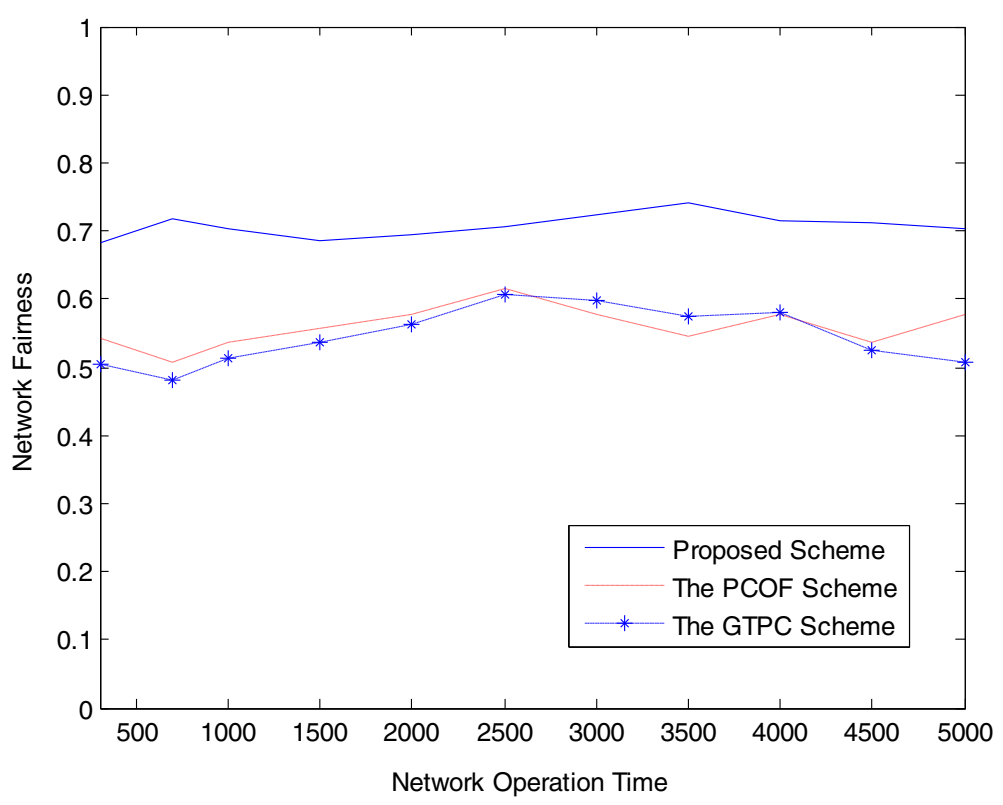

Figure 3 Network fairness.

tremendous increasing wireless capacity. During femtocell network operations, appropriate power control strategy is a crucial issue to enhance the network performance. In this article, we propose a new fair-efficient power control scheme based on the WVG model. By using this dynamics of feedback loop, FAPs and users can be interacting with one another and adaptively make their decisions. Under rapidly changing network environments, the proposed voting game-based approach is an effective way to reach a globally desirable network performance between two conflicting objectives-efficiency and fairness. From simulation results, we can confirm that the proposed power control scheme outperforms existing schemes in terms of system throughput, user payoff, network fairness, etc.

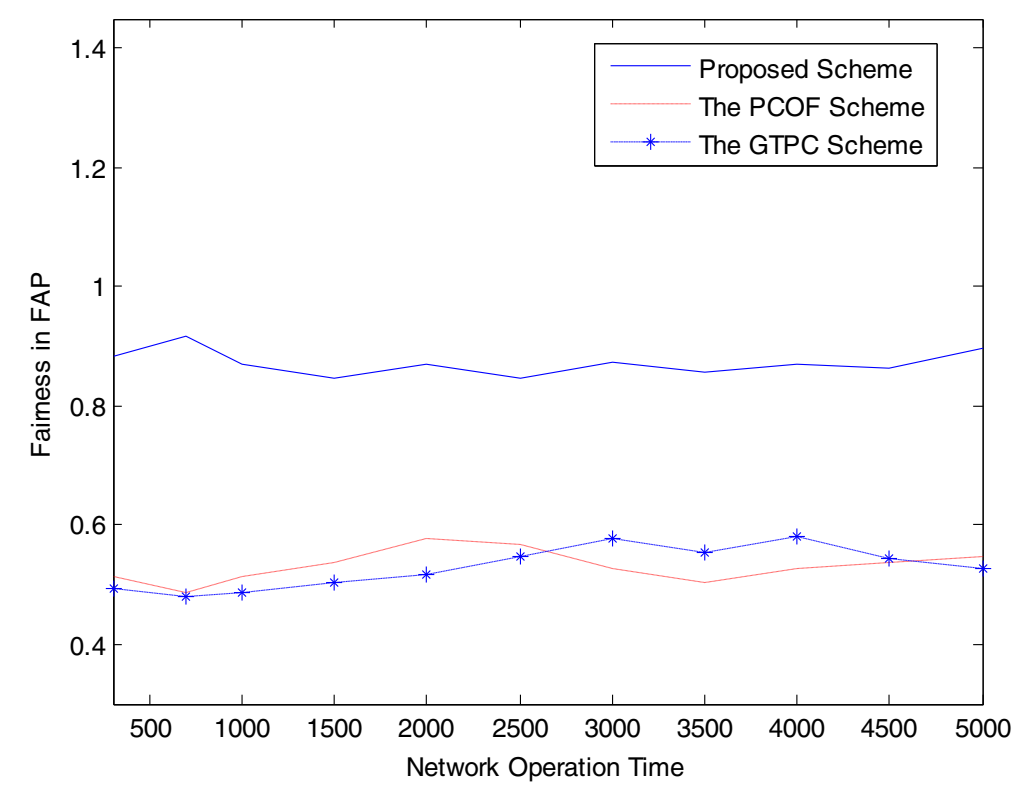

Figure 4 Fairness in FAP. 


\section{Competing interests}

The authors declare that they have no competing interests.

\section{Acknowledgment}

This research was supported by the Basic Science Research Program through the National Research Foundation of Korea (NRF) funded by the Ministry of Education, Science and Technology (2012-0005191) and by the Sogang University Research Grant of 201110011.

Received: 17 May 2012 Accepted: 31 January 2013

Published: 21 February 2013

\section{References}

1. V. Chandrasekhar, J. Andrews, A. Gatherer, Femtocell networks. IEEE Commun. Mag. 46, 59-67 (2008)

2. M.Z. Chowdhury, B. Minh Trung, J. Yeong Min, Neighbor cell list optimization for femtocell-to-femtocell handover in dense femtocellular networks. Int. Conf. Ubiquitous Future Networks (ICUFN'11) 1, 241-245 (2011)

3. L. Yizhe, F. Zhiyong, Enterprise femtocell network optimization based on neural network modeling. IEEE CCNC'11 1, 1130-1131 (2011)

4. M. Dianati, X. Shen, S. Naik, A new fairness index for radio resource allocation in wireless networks. Proc. IEEE WCNC 2, 712-715 (2005)

5. J. Mo, J. Walrand, Fair end-to-end window-based congestion control. IEEE/ ACM Trans.Networking 8, 556-567 (2000)

6. Y. Bachrach, R. Meir, M. Zuckerman, J. Rothe, J.S. Rosenschein, The cost of stability in weighted voting games. AAMAS'09 2, 1289-1290 (2009)

7. J. Leino, Applications of game theory in ad hoc networks. Master's Thesis (Helisnki University of Technology, Helisnki, 2003)

8. V. Srivastava, J. Neel, A.B. MacKenzie, R. Menon, L.A. DaSilva, J.E. Hicks, J.H. Reed, R.P. Gilles, Using game theory to analyze wireless ad hoc networks. IEEE Commun. Surv. Tutorials 7, 46-56 (2005)

9. B. Yoram, E. Edith, Divide and conquer: false-name manipulations in weighted voting games. Int. Conf. Autonomous Agents Multiagent Syst. (AAMAS'08) 2, 975-982 (2008)

10. M. Zuckerman, P. Faliszewski, Y. Bachrach, E. Elkind, Manipulating the quota in weighted voting games. AAAl'08 Proc. 23rd National Conf Artif. Intelligence 1, 215-220 (2008)

11. D. Lopez-Perez, A. Valcarce, G. De La Roche, E. Liu, J. Zhang, Access methods to wimax femtocells: a downlink system-level case study. Commun. Syst. 2008. ICCS 2008. 11th IEEE Singapore International Conference 1, 1657-1662 (2008)

12. A. Valcarce, G.D.L. Roche, A. Jüttner, D. López-Pérez, J. Zhang, Applying FDTD to the coverage prediction of WiMAX femtocells. EURASIP J. Wirel. Commun. Netw. 2009 (2009). Article no. 3

13. D. Lopez-Perez, G. de la Roche, A. Valcarce, A. Juttner, J. Zhang, Interference avoidance and dynamic frequency planning for wimax femtocells networks. Commun. Syst. 2008. ICCS 2008. 11th IEEE Singapore International Conference 1, 1579-1584 (2008)

14. K. Sundaresan, S. Rangarajan, Efficient resource management in OFDMA femto cells. MobiHoc'09 ACM international symposium on Mobile ad hoc networking and computing 1, 33-42 (2009)

15. C.H. Ko, H.Y. Wu, On-demand resource-sharing mechanism design in twotier OFDMA femtocell networks. IEEE Trans. Vehicular Technol. 60, 1059-1071 (2011)

16. S.Y. Lien, Y.Y. Lin, K.C. Chen, Cognitive and game-theoretical radio resource management for autonomous femtocells with QoS guarantees. IEEE Trans. Wirel. Commun. 10, 2196-2206 (2011)

17. A. Attar, V. Krishnamurthy, O.N. Gharehshiran, Interference management using cognitive base-stations for UMTS LTE. IEEE Commun. Mag. 49, 152-159 (2011)

18. G.J. Foschini, Z. Miljanic, A simple distributed autonomous power control algorithm and its convergence. IEEE Trans. Veh. Technol. 42, 641-646 (1993)

19. X. Li, L. Qian, D. Kataria, Downlink power control in co-channel macrocell femtocell overlay. Inf. Sci. Syst.'09 (CISS'09) 1, 383-388 (2009)

20. E.J. Hong, S.Y. Yun, C. Dong-Ho, Decentralized power control scheme in femtocell networks: a game theoretic approach. IEEE Proc. Personal, Indoor and Mobile Radio Commun. 1, 415-419 (2009)

21. F. Meshkati, H.V. Poor, S.C. Schwartz, R.V. Balan, Energy-efficient power and rate control with QoS constraints: a game-theoretic approach. IWCMC $1,1435-1440(2006)$

22. C. Long, Q. Zhang, B. Li, H. Yang, X. Guan, Non-cooperative power control for wireless ad hoc networks with repeated games. IEEE J. Sel. Areas Commun. 25, 1101-1112 (2007)

23. S. Kim, An adaptive online power control scheme based on the evolutionary game theory. IET Commun. 5(18), 2648-2655 (2011)

doi:10.1186/1687-1499-2013-44

Cite this article as: Kim: Femtocell network power control scheme

based on the weighted voting game. EURASIP Journal on Wireless

Communications and Networking 2013 2013:44

\section{Submit your manuscript to a SpringerOpen ${ }^{\odot}$ journal and benefit from:}

- Convenient online submission

Rigorous peer review

- Immediate publication on acceptance

- Open access: articles freely available online

- High visibility within the field

- Retaining the copyright to your article

Submit your next manuscript at $>$ springeropen.com 